\title{
Quantum coherence of multiqubit states in correlated noisy channels
}

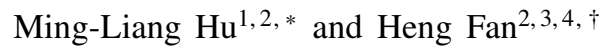 \\ ${ }^{1}$ School of Science, Xi'an University of Posts and Telecommunications, Xi'an 710121, China \\ ${ }^{2}$ Institute of Physics, Chinese Academy of Sciences, Beijing 100190, China \\ ${ }^{3}$ CAS Center for Excellence in Topological Quantum Computation, \\ University of Chinese Academy of Sciences, Beijing 100190, China \\ ${ }^{4}$ Songshan Lake Material Laboratory, Dongguan 523000, China
}

\begin{abstract}
The long-time maintenance of quantum coherence is crucial for its practical applications. We explore decoherence process of a multiqubit system passing through a correlated channel (phase flip, bit flip, bit-phase flip, and depolarizing). The results show that the decay of coherence was evidently delayed when the consecutive actions of the channel on the sequence of qubits has some classical correlations. In particular, the relative entropy of coherence for a system with large number of qubits is more robust than that with small number of qubits. We also provide an explanation for the delayed decoherence by exploring the interplay between the change of the unlocalized quantum coherence and the total correlation gain of the multiqubit system.

PACS numbers: 03.67.Mn, 03.65.Ta, 03.65.Yz
\end{abstract}

\section{INTRODUCTION}

Quantum coherence originates from the superposition principle of states. It captures an essential nature of quantum systems which is different from the classical coherence phenomena [1]. Due to this nature, quantum coherence attracted wide interests of researchers [2, 3]. One of such interest is to seek general conditions under which this genuine quantum property can be sustained for a system subject to typical sources of noise. The reasons for such an interest is that quantum coherence can be exploited as a resource for many quantum information and communication tasks [4]. Recent studies also provide evidence that the long-lived quantum coherence is crucial for defeating the classical limits of measurement accuracy in quantum metrology [5-7] and for enhancing certain biological processes [8]. But in practice, the unavoidable interaction of a principal system with its surroundings results in decoherence in most situations. As a consequence, the advantage of quantum coherence to empower the performance of quantum tasks will disappear in a very short time interval, and this remains one of the major obstacles for its wide applications.

Due to the above reasons, making clear decoherence mechanism of a principal system in the presence of typical noises becomes one of the major goals for accomplishing new quantum technologies. In 2014, Baumgratz et al. [9] introduced a rigorous framework for quantifying coherence and defined the $l_{1}$ norm of coherence and relative entropy of coherence. Subsequently, many other faithful coherence measures such as the entanglement-based coherence measures [10], the robustness of coherence [6, 11], and the convex roof measures of coherence [12-14], have also been introduced. All these paved the way for a quantitative analysis of the decoherence process of a system coupled to its surrounding environments or under the action of specific quantum channels.

Based on the measures of coherence, much endeavors have

*Electronic address: mingliang0301@163.com

†Electronic address: hfan@iphy.ac.cn been devoted to clarifying decoherence mechanism of an open quantum system. The attempts to achieve this goal were carried out mainly along two different but closely related directions. The first one is aimed at explaining role of active quantum operations on coherence of a state [15-19], the cohering and decohering power of specific quantum channels [20-25], the coherence-breaking channels [26], and the non-coherencegenerating channels [27]. There are also some works concentrating on the steered coherence at one party of a bipartite state by local operations on its another party [28-31]. The second direction is focused on exploring coherent properties of a principal system coupled to environment. Along this direction, the frozen phenomenon of coherence $[32-35]$ and the factorization relation for coherence evolution [36] have been observed. Several theoretical studies focused on suppressing the undesirable decay of coherence [37-39] or generating steady-state coherence [40, 41] have also been performed.

While the above works studied only the case for which the channel acts identically and independently on the sequence of qubits passing through it, the effects of correlations on consecutive applications of the channel on coherence of these qubits is usually neglected. But this is indeed a very realistic problem which should be taken into account in experiments. For example, when $N$ qubits traverse a channel, apart from the very special case for which the time interval between its successive applications on the qubits is infinitely small, the channel will has partial memory about its past history and the uncorrelated channel model will not be applicable [42]. Especially, the correlated applications of the channel and the temporal correlations in the evolution process of each qubit may together affect decoherence process of a multiqubit system.

In this paper, we make a step toward the above problem. We consider a model with classical correlations between consecutive applications of a channel [43], and examine how the coherence of a multiqubit state changes when one varies correlation strength of the channel. This can help to confirm potential role of the classical correlation of a correlated channel on suppressing decoherence. We also explore interplay between the extra correlations created by the channel and change of unlocalized quantum coherence for the principal system, aimed at 
providing an explanation for the delayed decoherence. As any physical process (time evolution, quantum operation, etc.) can be represented as a channel transforming the input states into output ones, the decohering channel model considered here is rather general, and the obtained results are hoped to shed some light on understanding decoherence process of a multipartite system immersed in real environments.

\section{PRELIMINARIES}

We start by collecting some preliminaries that we employ in this paper. For the purpose of providing a comparative study, we use two different coherence measures. The first one is the $l_{1}$ norm of coherence defined as [9]

$$
C_{l_{1}}(\rho)=\min _{\delta \in \mathcal{I}}\|\rho-\delta\|_{l_{1}}=\sum_{i \neq j}|\langle i|\rho| j\rangle|,
$$

where $\mathcal{I}$ denotes the set of incoherent states which are diagonal in the prefixed reference basis $\{|i\rangle\}$.

The second measure we adopt is the relative entropy of coherence given by [9]

$$
C_{r}(\rho)=\min _{\delta \in I} S(\rho \| \delta)=S(\Delta(\rho))-S(\rho),
$$

with $\Delta(\rho)=\sum_{i}\langle i|\rho| i\rangle|i\rangle\langle i|$ denoting the full dephasing of $\rho$ in the basis $\{|i\rangle\}$, and $S(\cdot)$ is the von Neumann entropy.

Next, we recall the model characterizing correlated channel that we intend to adopt [43]. It provides a proper mathematical tool apt to describe classical correlations between consecutive applications of the channel on a sequence of quantum systems [42]. For a single-qubit state $\rho_{0}$, the common action of a channel $\mathcal{E}$ on it can be described by a random rotation of it. That is, $\rho=\sum_{i=0}^{3} E_{i} \rho_{0} E_{i}^{\dagger}$, where the Kraus operators $E_{i}=\sqrt{p_{i}} \sigma_{i}$, with $\sigma_{0}=\mathbb{1}$ being the identity operator, $\sigma_{1,2,3}$ being the Pauli operators, and $p_{i}$ constitute a probability distribution. Similarly, if $\rho_{0}$ is a $N$-qubit state and $\mathcal{E}$ acts identically and independently on each of the qubit, the output state will be given by

$$
\rho=\sum_{i_{1} i_{2} \cdots i_{N}} E_{i_{1} i_{2} \cdots i_{N}} \rho_{0} E_{i_{1} i_{2} \cdots i_{N}}^{\dagger},
$$

where $E_{i_{1} i_{2} \cdots i_{N}}=\sqrt{p_{i_{1} i_{2} \cdots i_{N}}} \sigma_{i_{1}} \otimes \sigma_{i_{2}} \otimes \cdots \otimes \sigma_{i_{N}}$, and $p_{i_{1} i_{2} \cdots i_{N}}=$ $p_{i_{1}} p_{i_{2}} \cdots p_{i_{N}}$ represents the joint probability that a random sequence of Pauli rotations around the $i_{1} \cdots i_{N}$ axes is applied to the sequence of $N$ qubits traversing the channel.

Apart from the above case, one may encounters the case for which there are classical correlations on consecutive applications of the channel, and this may modify the way it acts on an input state. To be explicit, the action of the channel on a qubit may affects probability of its action on the subsequent qubits [42]. Macchiavello and Palma [43] proposed such a model, for which the joint probability is given by

$$
p_{i_{1} i_{2} \cdots i_{N}}=p_{i_{1}} p_{i_{2} \mid i_{1}} \cdots p_{i_{N} \mid i_{N-1}},
$$

with $p_{i_{n}} i_{n-1}=(1-\mu) p_{i_{n}}+\mu \delta_{i_{n} i_{n-1}}$. Here, $\mu \in[0,1]$ is a parameter which can be interpreted as the probability that the same Pauli transformation is applied to the qubits $i_{n-1}$ and $i_{n}$. For $\mu=0$, this model describes independent applications of the channel, while for $\mu=1$, the applications of the channel turn to be fully correlated [43].

In this paper, we focus our attention on the bit flip, bit-phase flip, phase flip, and depolarizing channels, which belong to the class of Pauli channels described by Eq. (3). The nonzero elements of $\left\{p_{i}\right\}$ for the former three channels can be represented by $p_{0}=1-p$ and $p_{i}=p$ (with $i=1,2$, and 3, respectively), while those for the last one are represented by $p_{0}=1-p$ and $p_{1,2,3}=p / 3$ [42-44].

\section{QUANTUM COHERENCE IN CORRELATED QUANTUM CHANNELS}

We take the maximally coherent state $\left|\Psi_{N}\right\rangle=\left|\Psi_{1}\right\rangle^{\otimes N}$ as the input of the channel, where $\left|\Psi_{1}\right\rangle=(|0\rangle+|1\rangle) / \sqrt{2}$. Due to possible memory effects arising dynamically during the evolution of each qubit and the memory effects induced by correlated actions of the channel [44], rich phenomena of decoherence process may be observed. For the purpose of comparing decoherence rate of the system with different number of qubits, we use in the following the normalized version of coherence

$$
\tilde{C}_{\alpha}(\rho)=\frac{C_{\alpha}(\rho)}{C_{\alpha}\left(\left|\Psi_{N}\right\rangle\right)}
$$

with $\alpha=\left\{l_{1}, r e\right\}, C_{l_{1}}\left(\left|\Psi_{N}\right\rangle\right)=2^{N}-1$, and $C_{r e}\left(\left|\Psi_{N}\right\rangle\right)=N$.

\section{A. Phase flip channel}

First, we consider the $l_{1}$ norm of coherence. For the uncorrelated and fully correlated phase flip channels, we have

$$
\tilde{C}_{l_{1}}(\rho)= \begin{cases}\frac{\sum_{n=1}^{N} \alpha_{n}^{(N)}|1-2 p|^{n}}{2^{N}-1} & \text { for } \mu=0, \\ \frac{2^{N-1}(1+|1-2 p|)-1}{2^{N}-1} & \text { for } \mu=1,\end{cases}
$$

where the coefficients $\alpha_{1}^{(N)}=N, \alpha_{N}^{(N)}=1$, and $\alpha_{n}^{(N)}=\alpha_{n-1}^{(N-1)}+$ $\alpha_{n}^{(N-1)}$ for $n \in[2, N-1]$.

Based on Eq. (6), one can see that $\tilde{C}_{l_{1}}(\rho)$ is symmetric with respect to $p=0.5$. For both the cases $p=0$ and $1, \tilde{C}_{l_{1}}(\rho)=1$, while for $p=0.5, \tilde{C}_{l_{1}}(\rho)$ takes its minimum which is zero for $\mu=0$ and finite for $\mu=1$. In the region of $p \leqslant 0.5, \tilde{C}_{l_{1}}(\rho)$ decreases with the increasing $p$. Moreover, for the uncorrelated channel $(\mu=0)$ with $p \neq\{0,1\}, \tilde{C}_{l_{1}}(\rho)$ decreases with the increasing number $N$ of qubits and vanishes when $N \rightarrow \infty$. See, e.g., the dashed line displayed in Fig. 1 a). For the fully correlated channel $(\mu=1)$, however, $\tilde{C}_{l_{1}}(\rho)$ turns to be increased from $|1-2 p|$ to $(1+|1-2 p|) / 2$ when $N$ increases from 1 to $\infty$. In particular, from Fig. 11(d) one can observe that the lines of $\tilde{C}_{l_{1}}(\rho)$ with $N=7$ and $N \rightarrow \infty$ are already nearly overlapped. This indicates that the fully correlated actions of the phase flip channel can significantly enhance the $l_{1}$ norm of coherence in the whole time evolution process. 

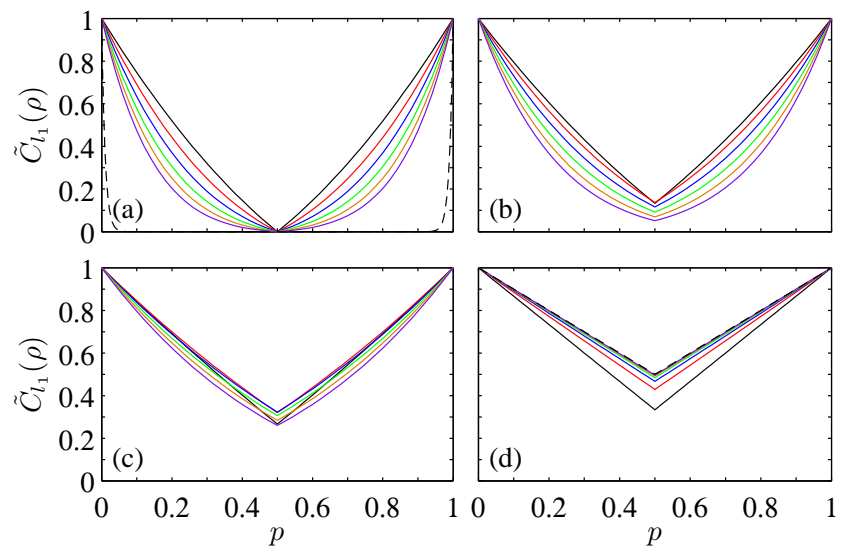

FIG. 1: The $p$ dependence of $\tilde{C}_{l_{1}}(\rho)$ for the input state $\left|\Psi_{N}\right\rangle$ and the correlated phase flip channel with (a) $\mu=0$, (b) $\mu=0.4$, (c) $\mu=0.8$, and (d) $\mu=1$. The solid black, red, blue, green, orange, and purple lines correspond to $N=2,3,4,5,6$, and 7, respectively. The dashed lines in (a) and (d) correspond to $N=100$ and $N \rightarrow \infty$, respectively.

For the intermediate value $\mu=0.5$ and $p \leqslant 0.5$ [for $p>0.5$, $\tilde{C}_{l_{1}}(\rho)$ can be obtained by substituting $p$ with $1-p$ ], we obtain

$$
\tilde{C}_{l_{1}}(\rho)=\frac{\sum_{n=1}^{N+1}(-1)^{n-1} \beta_{n}^{(N)} p^{n-1}}{2^{N}-1}
$$

with $\beta_{1}^{(N)}=2^{N}-1, \beta_{2}^{(N)}=2^{N-1}(N+1)$, and $\beta_{n}^{(N)}=2 \beta_{n}^{(N-1)}+$ $\beta_{n-1}^{(N-1)}$ for $n \in[3, N+1]$. For any fixed $N, \tilde{C}_{l_{1}}(\rho)$ decreases monotonically when $p$ increases from 0 to 0.5 , and apart from $p=\{0,1\}, \tilde{C}_{l_{1}}(\rho) \rightarrow 0$ when the qubit number $N \rightarrow \infty$. For general values of $\mu$, we performed numerical calculation. The results show that the dependence of $\tilde{C}_{l_{1}}(\rho)$ on $p$ is qualitatively the same to that of $\mu=0.5$. For fixed $p, \tilde{C}_{l_{1}}(\rho)$ decreases with the increasing $N$ for relative small $\mu$, see Fig. 1 b). But as can be seen from Fig. 11(c), $\tilde{C}_{l_{1}}(\rho)$ may does not always behave as a monotonic function of $N$ for relative large $\mu$.

From the above analysis one can see that correlated actions of the phase flip channel is beneficial for long-time preservation of the $l_{1}$ norm of coherence. We have performed numerical calculations with different $p$, and found that for the input state $\left|\Psi_{N}\right\rangle, \tilde{C}_{l_{1}}(\rho)$ always behaves as a monotonic increasing function of $\mu$. For $p=0.5$, we managed to obtain its analytical solution as

$$
\tilde{C}_{l_{1}}(\rho)=\frac{1}{2^{N}-1} \sum_{n=1}^{N-1} \eta_{n}^{(N)} \mu^{n}
$$

where $\eta_{1}^{(N)}=N-1, \eta_{N-1}^{(N)}=1$, and $\eta_{n}^{(N)}=\eta_{n-1}^{(N-1)}+\eta_{n}^{(N-1)}$ for $n \in[2, N-2]$. By using this equation, one can confirm again that $\tilde{C}_{l_{1}}(\rho)$ is a monotonic increasing function of $\mu$. That is, the $l_{1}$ norm of coherence can be enhanced by introducing classical correlation to this channel. But apart from the special case $\mu=$ 1 for which $\tilde{C}_{l_{1}}(\rho)$ always takes a finite value, the enhancement will becomes smaller and smaller with the increasing number $N$ of qubits, and $\tilde{C}_{l_{1}}(\rho) \rightarrow 0$ when $N \rightarrow \infty$. Further numerical calculation shows that the similar phenomena also happen for general values of $p \neq\{0,1\}$.
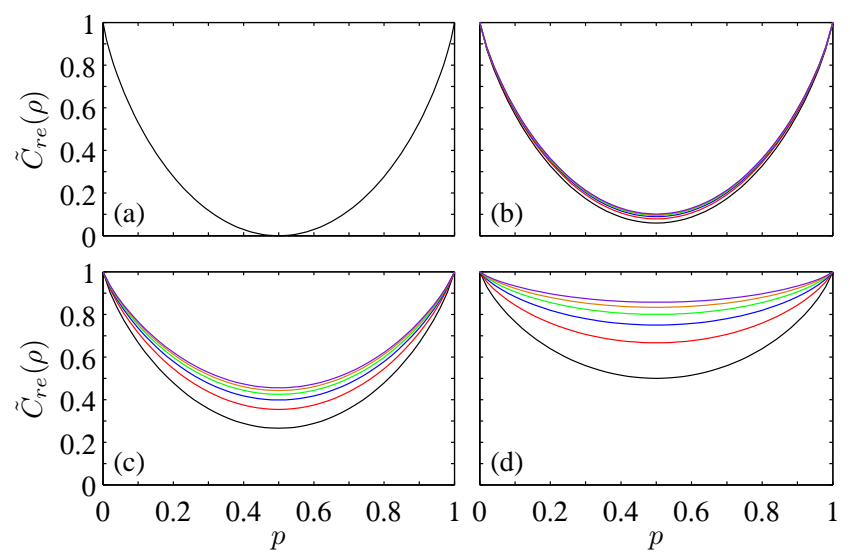

FIG. 2: The $p$ dependence of $\tilde{C}_{r e}(\rho)$ for the input state $\left|\Psi_{N}\right\rangle$ and the correlated phase flip channel with (a) $\mu=0$, (b) $\mu=0.4$, (c) $\mu=0.8$, and (d) $\mu=1$. The solid black, red, blue, green, orange, and purple lines correspond to $N=2,3,4,5,6$, and 7, respectively.

Next, we turn to consider the relative entropy of coherence. For the uncorrelated and fully correlated phase flip channels, analytical expressions of $\tilde{C}_{r e}(\rho)$ can be obtained as

$$
\tilde{C}_{r e}(\rho)= \begin{cases}1-H_{2}(p) & \text { for } \mu=0, \\ 1-\frac{1}{N} H_{2}(p) & \text { for } \mu=1,\end{cases}
$$

with $H_{2}(\cdot)$ being the binary Shannon entropy. $\tilde{C}_{r e}(\rho)$ decreases monotonically when $p$ increases from 0 to 0.5 , see Fig. 2 a) and (d). For $\mu=0, \tilde{C}_{r e}(\rho)$ is independent of the number $N$ of qubits, while for $\mu=1$, it increases with the increase of $N$ and approaches asymptotically to its maximum 1 when $N \rightarrow \infty$. This is a surprising phenomenon. It indicates that the relative entropy of coherence for a system with large number of qubits has an intrinsic rigidity against the fully correlated phase flip channel. This phenomenon is also in big contrast to that of the $l_{1}$ norm of coherence, as apart from the trivial cases $p=0$ and 1 , the latter can only approaches to a finite value other than the maximum 1 in the limit of $N \rightarrow \infty$.

For $0<\mu<1$, a further analysis shows that $\tilde{C}_{r e}(\rho)$ exhibits a linear dependence on $1 / N$. To be explicit, we have

$$
\tilde{C}_{r e}(\rho)=1-H_{2}(p)+k H_{2}(p)\left(1-\frac{1}{N}\right),
$$

and the factor $k$ can be evaluated analytically as

$$
k=\frac{\sum_{n} \epsilon_{n} \log _{2} \epsilon_{n}+2 H_{2}(p)}{H_{2}(p)} .
$$

where $\epsilon_{1,2}=p(1-p)(1-\mu), \epsilon_{3}=p(p+\mu-p \mu)$, and $\epsilon_{4}=$ $(1-p)(1-p+p \mu)$ are the eigenvalues of $\mathcal{E}\left(\left|\Psi_{2}\right\rangle\right)$.

From Eq. (10) one can verify directly that the $p$ dependence of $\tilde{C}_{r e}(\rho)$ is qualitatively the same to that of $\mu=1$. That is, it decreases monotonically when $p$ increases from 0 to 0.5 , see the exemplified plots displayed in Fig. 2(b) and (c). Moreover, $\tilde{C}_{r e}(\rho)$ increases monotonically with the increase of $\mu$, 


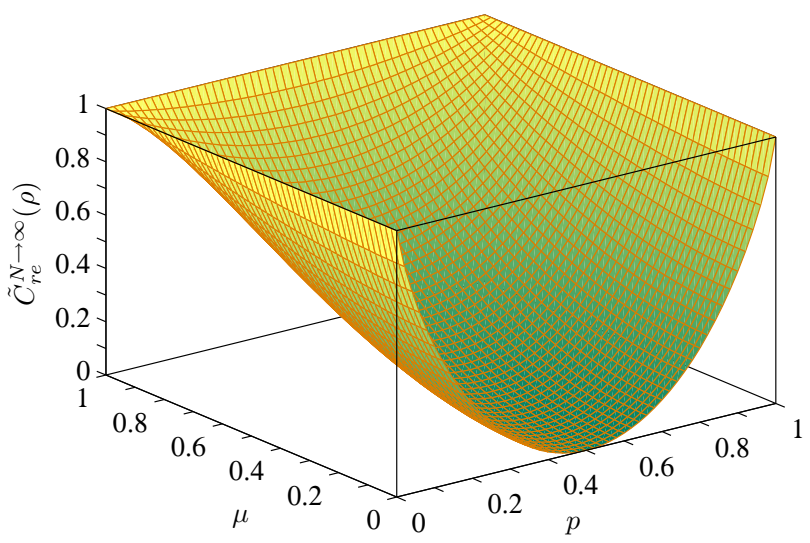

FIG. 3: The $p$ and $\mu$ dependence of $C_{r e}^{N \rightarrow \infty}(\rho)$ for the input state $\left|\Psi_{N}\right\rangle$ and the correlated phase flip channel.

i.e., the decay of the relative entropy of coherence can be suppressed evidently by the classical correlations arising from the consecutive applications of the phase flip channel.

From Eq. (10) one can also see that $\tilde{C}_{r e}(\rho)$ always increases with the increasing qubit number $N$. This is an important feature different from that exhibited by the $l_{1}$ norm of coherence. It shows again that the relative entropy of coherence with large number of qubits is more robust than that with small number of qubits, and reflects from one aspect the difference between these two measures of coherence [45]. When $N \rightarrow \infty, \tilde{C}_{r e}(\rho)$ approaches to its asymptotic value $C_{r e}^{N \rightarrow \infty}(\rho)$. This asymptotic value increases from $1-H_{2}(p)$ to its maximum 1 when $\mu$ increases from 0 to 1 (see Fig. 3). More importantly, $C_{r e}^{N \rightarrow \infty}(\rho)$ is finite apart from the very special point $(\mu, p)=(0,0.5)$. This further confirms our finding that the relative entropy of coherence for a system with large number of qubits has an intrinsic rigidity against the correlated phase flip channel.

Before ending this section, we give a short comment of the facts that when $N \rightarrow \infty, \tilde{C}_{l_{1}}(\rho) \rightarrow(1+|1-2 p|) / 2$ and $\tilde{C}_{r e}(\rho) \rightarrow$ 1 for $\mu=1$, while $\tilde{C}_{l_{1}}(\rho) \rightarrow 0$ and $\tilde{C}_{r e}(\rho) \rightarrow 1+(k-1) H_{2}(p)$ for $0<\mu<1$. At first glance, it seems to be contradictory, as any coherence measure vanishes for incoherent states and takes its maximum for maximally coherent states. But in fact there is no such a contradiction. This is because $\tilde{C}_{r e}(\rho) \rightarrow 1$ indicates $C_{r e}(\rho)$ is infinitely close to its maximum $N$, while the output state $\rho$ is still not maximally coherent. Similarly, $\tilde{C}_{l_{1}}(\rho) \rightarrow 0$ indicates $C_{l_{1}}(\rho)$ is far less than its maximum $2^{N}-1$, while the output state $\rho$ still maintains partial coherence.

\section{B. Bit flip, bit-phase flip, and depolarizing channels}

For these types of noisy channels, the considered coherence can be obtained directly based on the results for the correlated phase flip channel. We list them as follows:

(i) For the correlated bit flip channel, it is direct to obtain that $\left|\Psi_{N}\right\rangle$ is an eigenstate of $\sigma_{1}^{\otimes N}$ and, therefore, the $N$ qubits will pass undisturbed through this channel. As a consequence, the quantum coherence will frozen forever.

(ii) For the correlated bit-phase flip channel, as $\sigma_{2}=i \sigma_{1} \sigma_{3}$, the behaviors of coherence for the $N$ qubits will be completely the same to that of the $N$ qubits passing through the correlated phase flip channel.

(iii) For the correlated depolarizing channel, both $\tilde{C}_{l_{1}}(\rho)$ and $\tilde{C}_{r e}(\rho)$ can be obtained directly by substituting the parameter $p$ in the expressions of $\tilde{C}_{l_{1}}(\rho)$ and $\tilde{C}_{r e}(\rho)$ for the phase flip channel to $2 p / 3$, so their dynamical behaviors are qualitatively the same to that of the phase flip channel.

\section{TOTAL CORRELATION GAIN AND UNLOCALIZED QUANTUM COHERENCE}

In this section, we investigate the interplay between coherence of the $N$-qubit system and its correlation created by the correlated channel. It has been shown that unlocalized coherence, i.e., the coherence of a multipartite system subtract the coherence localized in each of its subsystem, captures a kind of correlation [46, 47]. For the input state $\left|\Psi_{N}\right\rangle$, there is no any correlation in it at the initial time. For $\mu=0$, from Eq. (9) one can obtain $C_{r e}(\rho)=\sum_{i} C_{r e}\left(\rho_{i}\right)$, with $\rho_{i}$ being the reduced density operator of the $i$ th qubit. Thus there is no correlation to be created in the $N$-qubit system when the phase flip channel is uncorrelated. But if $\mu>0$, the consecutive applications of the channel turn to be classically correlated and its correlation may be transferred to the $N$-qubit system. For example, when $\mu=1$ the unlocalized quantum coherence can be calculated as

$$
C_{r e}^{\mathrm{uqc}}(\rho)=C_{r e}(\rho)-\sum_{i} C_{r e}\left(\rho_{i}\right)=(N-1) H_{2}(p),
$$

and it is direct to show that $C_{r e}^{\mathrm{uqc}}(\rho)$ equals the quantum mutual information $I(\rho)=\sum_{i} S\left(\rho_{i}\right)-S(\rho)$, which characterizes the total correlation contained in a system [48].

In effect, for the input state $\left|\Psi_{N}\right\rangle$ and the four channels considered here, we always have $S(\Delta(\rho))=N$ and $S\left(\Delta\left(\rho_{i}\right)\right)=1$ $(\forall i)$, irrespective of the value of $\mu$. So $C_{r e}^{\text {uqc }}(\rho)=I(\rho)$, namely, $C_{r e}^{\text {uqc }}(\rho)$ equals the total correlation gain of the $N$ qubits after they passing through the correlated channel. One can further show that $C_{r e}^{\mathrm{uqc}}(\rho)$ is an increasing function of $\mu$. As $\mu$ quantifies the amount of classical correlation in the implementation of the channel [43], this result implies that the more classical correlation there is in the correlated channel, the more correlation will be transferred to the $N$-qubit system.

Moreover, if one concentrates only on the correlated phase flip channel, then for any multiqubit input state $\rho_{0}$, its diagonal part will remains unaffected. So one can obtain

$$
C_{r e}^{\mathrm{uqc}}(\rho)-C_{r e}^{\mathrm{uqc}}\left(\rho_{0}\right)=I(\rho)-I\left(\rho_{0}\right) .
$$

It indicates that the change of the unlocalized quantum coherence for the multiqubit system equals its total correlation gain. To be explicit, there are correlations created by the correlated channel in the multiqubit system only when $I(\rho)>I\left(\rho_{0}\right)$.

\section{CONCLUSION}

In conclusion, we have explored decoherence process of $N$ qubits when they pass through a correlated noisy channel. We 
used two coherence measures, i.e., the normalized $l_{1}$ norm of coherence and relative entropy of coherence, and considered four common noise sources: phase flip, bit flip, bit-phase flip, and depolarizing channels. Based on these, we analyzed in detail effects of the consecutive applications of the noisy channel on decoherence of the $N$-qubit system that is prepared initially in the maximally coherent state.

We observed here two distinctive phenomena which may be exploited for preserving coherence of a $N$-qubit system. First, both the decay of the $l_{1}$ norm of coherence and the relative entropy of coherence were suppressed if the consecutive actions of the channel has some correlations. Second, the two coherence measures show different dependence on qubit number $N$ of the system. The $l_{1}$ norm of coherence does not behave as a monotonic function of $N$, and apart from the situation that the channel is fully correlated, its value will becomes infinitesimal when $N \rightarrow \infty$. However, the relative entropy of coherence becomes more and more robust with the increase of $N$, and even when $N$ approaches infinite, it still maintains a considerable value. This indicates that the relative entropy of coherence for a system with large number of qubits has an intrinsic rigidity against detrimental effects of the channel.

To give an explanation for the delayed decoherence, we fur- ther explored the interplay between the unlocalized quantum coherence and the correlation gain of the $N$ qubits. The results show that for the input maximally coherent state and the four sources of noise considered here, the change of the unlocalized quantum coherence for the $N$ qubits equals their total correlation gain. Moreover, for the correlated phase flip channel, the change of the unlocalized quantum coherence equals the total correlation gain for arbitrary $N$-qubit input state. This implies that the delayed decay of coherence may be caused by the inflow of correlations from the channel to the system.

\section{ACKNOWLEDGMENTS}

This work was supported by National Natural Science Foundation of China (Grants No. 11675129, No. 91536108, and No. 11774406), National Key R \& D Program of China (Grants No. 2016YFA0302104 and No. 2016YFA0300600), the New Star Project of Science and Technology of Shaanxi Province (Grant No. 2016KJXX-27), the Strategic Priority Research Program of Chinese Academy of Sciences (Grant No. XDB28000000), and the New Star Team of XUPT.
[1] Z. Ficek and S. Swain, Quantum Interference and Coherence: Theory and Experiments, Springer Series in Optical Sciences (Springer, Berlin, 2005).

[2] A. Streltsov, G. Adesso, and M. B. Plenio, Rev. Mod. Phys. 89, 041003 (2017).

[3] M. L. Hu, X. Hu, J. C. Wang, Y. Peng, Y. R. Zhang, and H. Fan, Phys. Rep. 762-764, 1 (2018).

[4] M. A. Nielsen and I. L. Chuang, Quantum Computation and Quantum Information (Cambridge University Press, Cambridge, UK, 2000).

[5] V. Giovannetti, S. Lloyd, and L. Maccone, Science 306, 1330 (2004).

[6] C. Napoli, T. R. Bromley, M. Cianciaruso, M. Piani, N. Johnston, and G. Adesso, Phys. Rev. Lett. 116, 150502 (2016).

[7] K. Bu, U. Singh, S. M. Fei, A. K. Pati, and J. Wu, Phys. Rev. Lett. 119, 150405 (2017).

[8] N. Lambert, Y.-N. Chen, Y.-C. Cheng, C.-M. Li, G.-Y. Chen, and F. Nori, Nat. Phys. 9, 10 (2013).

[9] T. Baumgratz, M. Cramer, and M. B. Plenio, Phys. Rev. Lett. 113, 140401 (2014).

[10] A. Streltsov, U. Singh, H. S. Dhar, M. N. Bera, and G. Adesso, Phys. Rev. Lett. 115, 020403 (2015).

[11] M. Piani, M. Cianciaruso, T. R. Bromley, C. Napoli, N. Johnston, and G. Adesso, Phys. Rev. A 93, 042107 (2016).

[12] X. Yuan, H. Zhou, Z. Cao, and X. Ma, Phys. Rev. A 92, 022124 (2015).

[13] A. Winter and D. Yang, Phys. Rev. Lett. 116, 120404 (2016).

[14] X. Qi, T. Gao, and F. Yan, J. Phys. A 50, 285301 (2017).

[15] X. Hu, A. Milne, B. Zhang, and H. Fan, Sci. Rep. 6, 19365 (2016).

[16] Y. Yao, G. H. Dong, L. Ge, M. Li, and C. P. Sun, Phys. Rev. A 94, 062339 (2016).

[17] M. L. Hu, S. Q. Shen, and H. Fan, Phys. Rev. A 96, 052309 (2017).
[18] C. L. Liu, Y. Q. Guo, and D. M. Tong, Phys. Rev. A 96, 062325 (2017).

[19] M. Takahashi and E. Chitambar, J. Phys. A 51, 414003 (2018).

[20] A. Mani and V. Karimipour, Phys. Rev. A 92, 032331 (2015).

[21] P. Zanardi, G. Styliaris, and L. Campos Venuti, Phys. Rev. A 95, 052306 (2017).

[22] K. Bu, A. Kumar, L. Zhang, and J.Wu, Phys. Lett. A 381, 1670 (2017).

[23] G. Styliaris, L. Campos Venuti, and P. Zanardi, Phys. Rev. A 97, 032304 (2018).

[24] H. Situ and X. Hu, Quantum Inf. Process. 15, 4649 (2016).

[25] L. Zhang, Z. H. Ma, Z. H. Chen, and M. Fei, Quantum Inf. Process. 17, 186 (2018).

[26] K. Bu, Swati, U. Singh, and J. Wu, Phys. Rev. A 94, 052335 (2016).

[27] X. Hu, Phys. Rev. A 94, 012326 (2016).

[28] X. Hu and H. Fan, Sci. Rep. 6, 34380 (2016).

[29] D. Mondal, T. Pramanik, and A. K. Pati, Phys. Rev. A 95, 010301(R) (2017).

[30] M. L. Hu and H. Fan, Phys. Rev. A 98, 022312 (2018).

[31] M. L. Hu, X. M. Wang, and H. Fan, Phys. Rev. A 98, 032317 (2018).

[32] T. R. Bromley, M. Cianciaruso, and G. Adesso, Phys. Rev. Lett. 114, 210401 (2015).

[33] X. D. Yu, D. J. Zhang, C. L. Liu, and D. M. Tong, Phys. Rev. A 93, 060303(R) (2016)

[34] I. A. Silva et al., Phys. Rev. Lett. 117, 160402 (2016).

[35] A. Zhang, K. Zhang, L. Zhou, and W. Zhang, Phys. Rev. Lett. 121, 073602 (2018).

[36] M. L. Hu and H. Fan, Sci. Rep. 6, 29260 (2016).

[37] Y. J. Zhang, W. Han, Y. J. Xia, Y. M. Yu, and H. Fan, Sci. Rep. 5, 13359 (2015).

[38] X. B. Liu, Z. H. Tian, J. C. Wang, and J. L. Jing, Ann. Phys. 366, 102 (2016). 
[39] W. Wu and J. Q. Cheng, Quantum Inf. Process. 17, 300 (2018).

[40] G. Guarnieri, M. Kolář, and R. Filip, Phys. Rev. Lett. 121, 070401 (2018).

[41] C. Mukhopadhyay, Phys. Rev. A 98, 012102 (2018).

[42] F. Caruso, V. Giovannetti, C. Lupo, and S. Mancini, Rev. Mod. Phys. 86, 1203 (2014).

[43] C. Macchiavello and G. M. Palma, Phys. Rev. A 65, 050301(R) (2002).

[44] C. Addis, G. Karpat, C. Macchiavello, and S. Maniscalco, Phys.
Rev. A 94, 032121 (2016).

[45] C. L. Liu, X. D. Yu, G. F. Xu, and D. M. Tong, Quantum Inf. Process. 15, 4189 (2016).

[46] K. C. Tan, H. Kwon, C.-Y. Park, and H. Jeong, Phys. Rev. A 94, 022329 (2016).

[47] M. L. Hu and H. Fan. Phys. Rev. A 95, 052106 (2017).

[48] K. Modi, A. Brodutch, H. Cable, T. Paterek, and V. Vedral, Rev. Mod. Phys. 84, 1655 (2012). 